\title{
Análise de sistemas de informações utilizados como suporte para os processos de estimação de custos e formação de preços
}

\author{
Antônio Artur de Souza \\ Doutor em Management Sciences pela University of Lancaster \\ Rua Artur Itabirano, 99, São José, 31275-020 Belo Horizonte, MG \\ E-mail: artur@face.ufmg.br
}

Márcio Noveli

Mestre em Administração pela Universidade Federal do Paraná - UFPR Rua Pioneiro Virgílio Acelino Cardoso, 371, Vila Santa Isabel, 87080-270

E-mail: marcionoveli@yahoo.com.br

Maringá, PR

Ítalo Fernando Minello

Mestre em Administração pela Universidade Federal de Santa Catarina - UFSC Rua Otávio Miguel da Silva, 819, Apto. 32, 08673-060 Suzano/SP

E-mail: italominello@terra.com.br

João Otávio Montanha Endrici

Mestrando em Administração pela Universidade Estadual de Maringá - UEM Rua São João, 139, Bloco B, Apto 18, Zona Leste, 87030-200 Maringá, PR

E-mail: endrici@yahoo.com.br

Ewerton Alex Avelar

Graduando em Ciências Contábeis pela Universidade Federal de Minas Gerais - UFMG Rua Hermílio Alves,138, Apto. 3, Santa Tereza, 31010-070 Belo Horizonte, MG E-mail: ewerton@face.ufmg.br

\section{RESUMO}

O processo de formação de preços (FP), embora fundamental para as empresas, é de difícil condução, por implicar diversos fatores. Nas empresas de produção por encomenda (EPEs), o processo é ainda mais complexo, em razão da natureza peculiar de suas operações. Estas empresas oferecem um produto bastante customizado, e as especificações dos clientes orientam todo o seu processo produtivo. Usualmente, essas empresas concorrem pelos pedidos de fabricação. A primeira etapa para a FP consiste na estimação de custos (EC) para a produção. Custos estimados incorretamente levam a preços distorcidos. Além da EC, muitos fatores, internos e externos às empresas, devem ser avaliados para que as decisões sejam tomadas corretamente e para que possa ser estabelecido um preço competitivo. Portanto, os gerentes das EPEs necessitam de informações acuradas para que tomem suas decisões com segurança e precisão, levando em consideração todos os aspectos relevantes para a FP. Os 
Análise de sistemas de informações utilizados como suporte para os processos de estimação de custos e formação de preços

Antônio Artur de Souza, Márcio Noveli, Ítalo Fernando Minello, João Otávio Montanha Endrici, Ewerton Alex Avelar

principais objetivos da pesquisa descrita no presente artigo foram: (1) analisar os processos de tomada de decisão relacionados com EC e FP em EPEs; e (2) verificar se os sistemas de suporte à decisão usados para auxiliar os gerentes nesses processos são adequados e se há necessidades não supridas por esses sistemas.

Palavras-chave: Empresas de produção por encomenda. Formação de preços. Sistemas de suporte à decisão.

\section{An analysis of the information systems used to support the cost estimation and pricing processes}

\section{ABSTRACT}

Despite being crucial to all sorts of companies, the pricing process is difficult to be performed due to the several factors, which must be considered when defining prices. In make-to-order (MTO) companies, pricing is even more complex because of the peculiar nature of the transactions that these companies perform with their customers. The MTO companies offer highly customized products and usually compete among thenselves for the client's orders. The first stage of the pricing process is always the estimation of all the manufacturing costs. These costs, if incorrectly estimated, lead to inaccurate prices. Besides the cost estimates, many other factors must be evaluated in order to have the price decisions carried out correctly. These factors can be internal or external to the company's control. The evaluation of these factors is crucial to have prices that are competitive and compatible to the costs. Therefore, MTO companies need precise information so their managers can be able to make reliable decisions taking into consideration all relevant information to the definition of prices. The main objectives of the research described in this paper were (1) to analyze the decision-making process related to cost estimation and pricing, and (2) to verify if the decision support systems used by these companies to aid their managers are adequate and if there are information needs not fulfilled by such systems.

Key words: Make-to-order companies. Pricing process. Decision support systems.

\section{INTRODUÇÃO}

As empresas, de modo geral, encontram dificuldades para estabelecer o preço de venda de seus produtos. Encontrar o preço certo não é uma tarefa simples, pois é influenciada por diversos fatores, internos e externos à empresa, que, muitas vezes, são de difícil identificação e mensuração. Assim, não raramente, os profissionais responsáveis pelo processo de formação de preços (FP) delegam essa tarefa ao 
Análise de sistemas de informações utilizados como suporte para os processos de estimação de custos e formação de preços

Antônio Artur de Souza, Márcio Noveli, Ítalo Fernando Minello, João Otávio Montanha Endrici, Ewerton Alex Avelar

mercado, por serem incapazes de identificar e controlar todos os fatores necessários ao estabelecimento de seu próprio preço. É também usual empregarem métodos convencionais simples para realizá-la, os quais geralmente não levam em consideração todos os fatores relevantes ao processo.

No presente ambiente empresarial, altamente competitivo, delegar ao mercado a formação de seu preço, da mesma forma que usar métodos falhos para isso é, no mínimo, arriscado para uma empresa. A formação de um preço ideal para o produto aparenta ser imprescindível. Bruni e Famá (2004) afirmam que um preço equivocado de um produto ou serviço pode causar a ruína da empresa. Diante de afirmações como esta, sustentadas pela crescente agressividade do mercado globalizado, algumas empresas empenham-se em melhorar as metodologias usadas para a FP.

As empresas de produção por encomenda (EPEs) têm uma dificuldade ainda maior para formar seus preços, em razão das peculiaridades de seu processo produtivo. Usualmente, trabalham com produtos bastante customizados, e a principal referência para precificá-los são os custos no qual a empresa incorre para produzí-los. Sendo assim, o processo de estimação de custos (EC) é essencial para as EPEs. Estas precisam de informações acuradas sobre seus custos para uma estimativa correta, além de outras informações, internas e externas à empresa, para formar o preço ideal. Porém, essas informações são de difícil obtenção, e, geralmente, imprecisas, podendo levar os responsáveis pela EC e FP a dúvidas e erros. Uma alternativa que pode contribuir para a correção de tais falhas, consiste em usar um sistema de informações para suporte à decisão que leve em consideração todas as peculiaridades do processo produtivo das EPEs, que forneça informações confiáveis e que auxilie os responsáveis pelos processos de EC e FP na tomada de suas decisões.

Problemas relativos aos processos de EC e FP tendem a ser bastante desestruturados nas EPEs, fazendo com que o uso de sistemas de informações operacionais, tais como os Sistemas de Relatórios Gerenciais (SRGs) e os Sistemas de Processamento de Transações (SPTs), que se apresentam eficazes para auxiliar decisões referentes a problemas rotineiros e programados (ZWASS, 1992), se mostrem inapropriados para decisões referentes à EC e a FP. O uso de um Sistema de Suporte 
Análise de sistemas de informações utilizados como suporte para os processos de estimação de custos e formação de preços

Antônio Artur de Souza, Márcio Noveli, Ítalo Fernando Minello, João Otávio Montanha Endrici, Ewerton Alex Avelar

à Decisão (SSD) tem demonstrado ser uma alternativa viável para o auxílio no processo de tomada de decisão nesses casos, uma vez que este tipo de sistema é projetado para solucionar problemas desestruturados e de longo prazo.

O presente artigo apresenta os resultados de uma pesquisa que teve como principal objetivo analisar os sistemas de informações utilizados para auxiliar nos complexos processos de EC e FP em EPEs. A pesquisa foi realizada no período de 2002 a 2004 em EPEs da região noroeste do Paraná. A coleta de dados compreendeu observação não participante e pesquisa documental. A pesquisa de campo possibilitou reunir dados bastante relevantes para realizar a análise.

Nas seções 2 a 6, são apresentados os fundamentos teóricos de EPEs, FP, EC, necessidades de informações e SSDs, essenciais para a apresentação dos resultados da pesquisa descrita neste artigo. Na seção 7 destaca-se a metodologia utilizada para a condução da pesquisa. Nas seções 8 a 10, faz-se a análise dos resultados obtidos com a pesquisa. Na seção 8, apresenta-se uma caracterização dos processos de EC e FP em EPEs. Na seção 9 apresentam-se os fatores internos e externos, e a interdependência entre EC e FP. Na seção 10, apresentam-se os sistemas de informações utilizados para suporte à decisão referentes aos processos de EC e FP. $\mathrm{Na}$ seção 11 são apresentadas as conclusões da pesquisa.

\section{EMPRESAS DE PRODUÇÃO POR ENCOMENDA}

A pesquisa apresentada neste artigo trata somente das EPEs, ou seja, empresas com sistema de produção por ordem. Essas empresas produzem uma ampla variedade de produtos que geralmente diferem muito entre si, devido ao fato de serem elaborados sob encomenda dos clientes. Hansen e Mowen (2001) afirmam que a principal característica das empresas que possuem um sistema de produção por ordem é o fato dos custos dos produtos elaborados serem distintos para cada encomenda. Ao contrário das empresas que utilizam um sistema de produção contínua, onde os

produtos são bastante similares, elaborados fundamentalmente para estoque e os custos de elaboração de cada produto não sofrem, praticamente, qualquer alteração. 
Análise de sistemas de informações utilizados como suporte para os processos de estimação de custos e formação de preços

Antônio Artur de Souza, Márcio Noveli, Ítalo Fernando Minello, João Otávio Montanha Endrici, Ewerton

A proposta de classificação dos tipos de processos em operações de manufatura de Slack et al. (2002) mostra-se adequada para entender melhor o processo produtivo das EPEs. Segundo esses autores, tais processos podem ser divididos em cinco tipos distintos. Primeiro, Projeto, no qual o período para a confecção do produto é longo, as atividades produtivas podem ser alteradas durante o processo, a variedade da produção é grande e os recursos de transformação utilizados são, geralmente, específicos à produção de cada produto. Segundo, Processos de jobbing, que também lidam com alta variedade e baixo volume de produção, entretanto, produzem mais itens e, usualmente, menores que os processos de projeto, além de seus recursos de produção serem compartilhados entre diversos produtos. Terceiro, Processo de lotes ou bateladas, no qual, apesar de haver grande variedade na produção, os produtos são confeccionados em lotes. Quarto, Processo de produção em massa, em que há produção em alto volume e variedade relativamente baixa. Quinto, Processos contínuos, que possuem volume maior e variedade menor que os processos de produção em massa.

Os processos das EPEs são geralmente do tipo projeto ou jobbing, produtos com alto grau de diferenciação e baixo volume de produção. Como exemplos destes dois processos, pode-se citar: a) construção de navios, túneis, prédios (projetos); e b) alfaiates que trabalham por encomenda e gráficas que produzem diferentes ingressos para eventos sociais distintos (jobbing). Embora o mais comum sejam as EPEs pertencerem a setores da indústria, como o de metais pesados e algumas indústrias de móveis, muitas empresas prestadoras de serviços também trabalham por encomenda. Poderiam ser citadas as empresas de auditoria, de segurança, as organizadoras de eventos e as produtoras de filmes.

As EPEs trabalham atendendo às especificações dos clientes, que orientam todo o seu processo produtivo. Estes solicitam orçamentos e, frequentemente, realizam concorrências para identificar qual empresa contratarão para realizar o trabalho. Para ganhar os pedidos de fabricação, as EPEs têm de oferecer um preço abaixo do oferecido pelas concorrentes, porém acima de seus custos de fabricação, pois não é desejável para a empresa incorrer em prejuízos. 
Análise de sistemas de informações utilizados como suporte para os processos de estimação de custos e formação de preços

Antônio Artur de Souza, Márcio Noveli, Ítalo Fernando Minello, João Otávio Montanha Endrici, Ewerton Alex Avelar

\section{FORMAÇÃO DE PREÇOS}

Em sentido amplo, preço é a soma dos valores que os consumidores trocam pelo benefício de possuírem ou usarem um produto ou serviço (Kotler e Amstrong, 1999). Os objetivos dos preços podem ser assim definidos: sustentar a estratégia de posicionamento, atingir os objetivos financeiros propostos e ajustar a oferta à realidade de mercado. Dessa forma, as decisões relacionadas a preço podem ser consideradas como estratégicas para a empresa, e precisam ser embasadas em informações precisas.

As decisões relativas a preços podem ser consideradas como uma das mais difíceis enfrentadas pelos gerentes. Embora não seja o único aspecto de marketing a ser analisado, o preço é crucial para a prosperidade de qualquer empresa. Ele está intimamente relacionado aos seus lucros ou prejuízos, sucessos ou fracassos. Dolan e Simon (1998) afirmam que, geralmente, as principais dificuldades encontradas para a adoção de uma política de preços eficiente no atual contexto empresarial são: interdependência entre os produtos e os mercados; o fácil acesso a uma alta gama de informações por parte dos consumidores; e o contexto de crescente diversidade competitiva.

Kotler e Armstrong (1999) apresentam três metodologias normalmente utilizadas para a FP: a partir da concorrência; a partir dos custos de produção; e a partir do valor percebido pelo consumidor. Ressaltam, também, a relevância de diversos outros fatores que influenciam a FP e que não devem ser colocados à margem nesse processo, tais como: o tipo de mercado no qual a empresa se insere (concorrência perfeita, concorrência monopolista, concorrência oligopolista ou monopólio), e o posicionamento estratégico para o produto (sobrevivência, maximização do lucro, liderança de participação no mercado ou liderança de qualidade).

A maioria dos autores concorda que, independentemente da metodologia utilizada na FP, a análise de três fatores é condizente para a adequada precificação de um produto: concorrência, custos e valor percebido pelo consumidor. Kotler (2000) ressalta que a empresa só estará apta para selecionar o preço de venda de seu produto 
Análise de sistemas de informações utilizados como suporte para os processos de estimação de custos e formação de preços

Antônio Artur de Souza, Márcio Noveli, Ítalo Fernando Minello, João Otávio Montanha Endrici, Ewerton Alex Avelar

quando terminar a avaliação dos 3 Cs: programação da demanda de clientes, a função custo e os preços dos concorrentes.

Portanto, é facilmente perceptível que não existe uma fórmula mágica para a FP. Diversos fatores necessitam ser avaliados no estabelecimento do preço do produto ou serviço. Além disso, vários departamentos, cada um com suas prioridades, devem participar das decisões relativas à precificação, o que acaba, muitas vezes, gerando conflitos internos na empresa (Dolan e Simon, 1998).

Oferecendo um produto bastante customizado, que deve atender a uma demanda específica e que, muitas vezes, não foi anteriormente produzido pela empresa, as EPEs usam os próprios custos como principal referência na sua FP. "A maioria das empresas [de produção por ordem] calcula suas margens de lucro sobre cada serviço por meio de um mark-up, ou seja, calcula um percentual sobre os custos incorridos para determinar seu preço" (Atkinson et al., 2000, p. 251). Bruni e Famá (2004) apresentam o seguinte modelo para representar a relação entre gastos (incluídas as despesas), lucros e preço de venda para as EPEs: gastos somados ao lucro desejado é igual ao preço de venda do produto.

Verifica-se que nas EPEs os custos são essenciais para a tomada de decisão sobre o preço a ser cobrado pelo produto. Embora não seja o único fator a ser analisado, os custos são a principal referência para a FP. Portanto, estimá-los corretamente é fundamental. Como o processo de EC influencia diretamente a FP, o custo estimado deve ficar o mais próximo possível do real. Custos estimados de forma errada levam à sub ou à superavaliação do produto. Nesta, as EPEs perdem clientes por oferecer um preço maior que o dos concorrentes, e naquela podem incorrer em prejuízos ao longo da produção, pois, uma vez fixado o preço em contrato, não se pode mais negociá-lo com o cliente.

\section{ESTIMAÇÃO DE CUSTOS}

Custos estimados correspondem a aperfeiçoamentos, refinamentos e correções nos custos médios passados, em função de expectativas de mudanças. Custos 
Análise de sistemas de informações utilizados como suporte para os processos de estimação de custos e formação de preços

Antônio Artur de Souza, Márcio Noveli, Ítalo Fernando Minello, João Otávio Montanha Endrici, Ewerton Alex Avelar

estimados correspondem também a levantamentos de preços de materiais, serviços e dispêndios internos para a realização de trabalhos sem equivalentes históricos. Assim, estimar os custos significa prever os custos totais que ocorrerão se um determinado produto ou serviço for produzido. A idéia básica deste processo está em entender a relação entre os custos e as variáveis que os afetam.

A estimativa adequada dos custos é muito importante para as empresas. Maher (2001, p. 398) afirma que "estimativas precisas melhoram o processo de tomada de decisão; estimativas imprecisas resultam em ineficiências e aumentam a quantidade de decisões que não adicionam valor". Este autor apresenta as seguintes metodologias comumente usadas para estimar custos: Método das estimativas de engenharia - envolve a estimativa de custos com base na mensuração e precificação do trabalho associado a uma tarefa; Análise de contas - exige uma análise de cada conta que compõe o custo que está sendo considerado e sua classificação em fixo ou variável, dependendo da relação entre o custo e alguma atividade; Diagrama de estimativas alto-baixo (high-low) - estima os custos com base em duas observações de custos, geralmente correspondentes aos níveis de atividades mais alto e mais baixo; Métodos estatísticos (análise de regressão, normalmente) - consiste na aplicação de técnicas de regressão que objetivam gerar uma reta que melhor se ajuste a um conjunto de pontos representativos de dados sobre certas variáveis.

Outros métodos para EC podem ser citados: Método baseado na intuição e na experiência, utilizado pelos tomadores de decisão quando estão envolvidos em situações nas quais é relativamente fácil identificar o comportamento dos custos; Sistemas de estimação de custos computadorizados, que são, em síntese, sistemas de informações desenvolvidos pelas empresas para prever os custos de forma rápida e precisa; Sistemas de custeio de processo de múltiplos estágios (Atkinson et al., 2000), utilizados para determinar os custos do produto em indústrias de processamento de múltiplos estágios.

Nenhum desses métodos é totalmente eficaz. Todos possuem vantagens e desvantagens diante dos demais. Não obstante, alguns são melhores que outros, sendo que a verdadeira utilidade de um método para EC depende muito de quão bem o 
Análise de sistemas de informações utilizados como suporte para os processos de estimação de custos e formação de preços

Antônio Artur de Souza, Márcio Noveli, Ítalo Fernando Minello, João Otávio Montanha Endrici, Ewerton Alex Avelar

usuário do método conhece o negócio e os custos que estão sendo analisados. Além disso, vários fatores devem ser avaliados durante o processo de EC. Os mais relevantes são a curva de aprendizagem (relação sistêmica entre a experiência na realização de uma tarefa e o tempo necessário para realizá-la) e o sistema de custeio utilizado pela empresa.

Mesmo diante de várias metodologias e fatores que devem ser considerados para se realizar uma estimativa de custos, muitos dos profissionais responsáveis por esta tarefa, usualmente, dão maior relevância ao feeling e ao know how do que aos métodos científicos para executá-la. Essa atitude leva a imprecisões na EC. Sabendo que uma estimativa, após ser efetuada, influencia diversas decisões da empresa, invariavelmente, essas decisões podem não ser condizentes com a realidade.

\section{A NECESSIDADE DE INFORMAÇÕES DAS EMPRESAS DE PRODUÇÃO POR ENCOMENDA}

Estipular um preço adequado para cada produto nas EPEs é um processo bastante complexo. Isto ocorre, primeiramente, por causa da interdependência do processo de FP com o de EC (De Souza, 1995). A complexidade ocorre também devido à alta gama de fatores, internos e externos à empresa, que precisam ser levados em consideração nesses processos. Muitos deles são completamente novos, em consequência do atendimento às variadas especificações dos clientes. Para estabelecer um preço adequado, os tomadores de decisão nas EPEs precisam de informações precisas sobre todos os fatores relevantes que exercerão influência durante o processo de produção. Quanto mais precisas forem estas informações, menor será a necessidade dos profissionais utilizarem subjetivismo nas decisões, tornando-as mais coerentes.

Atualmente, a necessidade de informações adequadas no mundo empresarial é reconhecida como fundamental para a maioria dos autores, principalmente, no que se refere à FP. Dolan e Simon (1998) afirmam que um verdadeiro estrategista de preços dispõe de dados mais acurados, relevantes e específicos do que os administradores 
Análise de sistemas de informações utilizados como suporte para os processos de estimação de custos e formação de preços

Antônio Artur de Souza, Márcio Noveli, Ítalo Fernando Minello, João Otávio Montanha Endrici, Ewerton Alex Avelar

comuns. O profissional responsável pela EC e FP em EPEs necessita de informações fidedignas e pertinentes de forma rápida, concisa e sistematicamente organizadas, para tomar decisões corretas e formar o preço de maneira coerente e condizente com a realidade da empresa. Em suma, para determinar o preço adequado para seu produto, as EPEs precisam de informações precisas, no momento certo e disponibilizadas para os profissionais que as necessitam.

A competitividade e o aproveitamento das oportunidades que o ambiente proporciona estão diretamente relacionados à capacidade de captação, armazenamento, processamento e distribuição de informações. Dessa forma, parece vital à sobrevivência das empresas, agilidade e dinamicidade no processo de adequação às inovações provocadas pela turbulência do ambiente. Lawrence e Lorsch (1972) apresentam uma preocupação com o relacionamento das empresas inseridas num ambiente em rápida e permanente mutação. Essa preocupação consiste na busca da capacitação para o estabelecimento de um sistema criativo e flexível de solução de problemas, o que pode vir a facilitar a identificação e o aproveitamento de oportunidades potenciais nos processos de mudança. Sob esse enfoque, mudar as empresas parece ser uma questão de sobrevivência empresarial, particularmente no que se refere à geração e ao uso das informações, as quais podem proporcionar grande parte dessa capacitação.

Nesse sentido, os sistemas de informações computadorizados podem constituirse num suporte significativo para auxiliar os administradores no processo de capacitação e de tomada de decisão, contribuindo, assim, para facilitar a adaptação das empresas às constantes mudanças ambientais, através da disponibilidade e da rapidez de informações. Conforme Antonello (2004), a visão de mudança empresarial como algo planejado e intencional era compreensível em um contexto de poucas transformações ambientais, mas vivendo em um ambiente turbulento, as empresas passam a repensar a noção de ambiente estático e, para enfrentá-lo, necessitam criar sistemas de informações ágeis, flexíveis, eficientes e eficazes.

$O$ uso de um sistema de informações eficiente que auxilie os tomadores de decisão nas EPEs é uma alternativa para atenuar a complexidade dos processos de EC 
Análise de sistemas de informações utilizados como suporte para os processos de estimação de custos e formação de preços

Antônio Artur de Souza, Márcio Noveli, Ítalo Fernando Minello, João Otávio Montanha Endrici, Ewerton Alex Avelar

e FP. Diminui-se, assim, a necessidade de utilizar métodos baseados na intuição e na experiência para suprir a falta de informações, os quais são passíveis de imprecisões que podem trazer problemas para a empresa, devido ao crescente aumento da competitividade no mercado atual.

\section{SISTEMAS DE SUPORTE À DECISÃO}

Considerando-se que vivemos em uma época de intensa concorrência, mais do que vantagem competitiva, estar bem informado sobre seu ambiente mercadológico é condição de sobrevivência para as empresas modernas (Parente, 2003). Isso está intimamente relacionado ao sistema de informações de marketing que a empresa possui e sua utilização no processo decisório da mesma, pois neste sistema identificam-se as necessidades de informações, e desenvolvem-se informações através dos registros internos, da inteligência de marketing, da pesquisa de mercado e da análise da informação, estando sempre em contato com o ambiente de marketing, e, a partir disso, distribui-se as informações para a organização como um todo e seus dirigentes.

Neste sentido, este sistema de informações precisa estar adequadamente relacionado com todos os sistemas de informações da empresa, incluindo o sistema de informações contábeis e o sistema de informações de produção. A integração destes sistemas funcionais (setoriais) é necessária para a gestão empresarial e, no caso específico de EC e FP, é fundamental. As diferentes fontes para a utilização em SSDs são importantes em função do tipo de informações que são requeridas pelos tomadores de decisões, que está intimamente relacionado com o nível de tomada de decisão gerencial e o grau de complexidade das situações de decisão que eles enfrentam.

Segundo Zwass (1992), um SSD é desenvolvido para suportar diretamente o processo de decisão, estando apto para solucionar problemas, tanto estruturados como não estruturados, que podem ser facilmente programados. Seu principal objetivo é melhorar a efetividade da tomada de decisão nesses tipos de problemas. Geralmente, é utilizado quando o problema a ser solucionado é complexo ou quando a informação 
Análise de sistemas de informações utilizados como suporte para os processos de estimação de custos e formação de preços

Antônio Artur de Souza, Márcio Noveli, Ítalo Fernando Minello, João Otávio Montanha Endrici, Ewerton Alex Avelar

necessária para a tomada de decisão é de difícil obtenção. Enquanto os sistemas operacionais (tais como o SRG e o SPT) dizem respeito a decisões envolvendo problemas bem estruturados e de curto prazo, os SSDs, usualmente, referem-se a problemas relativamente não-estruturados e de longo prazo, sempre requerendo a participação de um ou mais gerentes (tomadores de decisão).

As seguintes características e capacidades dos SSDs são representadas por Turban (2004): a) oferecem suporte a vários níveis gerenciais, desde a cúpula executiva até a linha gerencial; b) oferecem suporte tanto para pessoas individuais quanto para grupos de trabalho; c) oferecem suporte para decisões interdependentes e ou sequenciais; d) envolvem todas as fases do processo de tomada de decisão (inteligência, design, escolha e implementação); e) com o tempo podem ser adaptados pelo usuário; f) geralmente, são fáceis de construir e usar; g) promovem o aprendizado; h) utilizam modelos quantitativos (padrão e/ou sob medida); i) podem ser disseminados por meio da Web; e j) permitem a realização de análises de sensibilidade.

Como mencionado anteriormente, em EPEs, a peculiaridade do processo produtivo dificulta os processos de EC e FP. Devido a diversos fatores, os problemas relativos a esses processos tendem a ser semi-estruturados ou desestruturados. Podese classificar como estruturado um problema rotineiro cuja forma de solucioná-lo é normalmente a mesma. O problema desestruturado refere-se a situações nas quais os procedimentos de solução não são conhecidos, ou seja, não é um problema rotineiro. Os problemas semi-estruturados têm operações bem conhecidas, mas também fatores incertos, que devem ser levados em consideração (Shimizu, 2001).

Muitas vezes, devido ao fato de a natureza das informações ser desestruturada, são cometidos erros e imprecisões na tomada de decisão, logo, a fim de tornar a tomada de decisão relativa aos processos de EC e FP mais precisa, a utilização de SSDs apresenta-se como uma alternativa viável. Um exemplo disso pode ser evidenciado na utilização de bancos de dados especializados, desenvolvidos pela empresa ou não, no que se refere a custos de produção, estabelecimento de preço, estimativas de custos, dentre outros. 
Análise de sistemas de informações utilizados como suporte para os processos de estimação de custos e formação de preços

Antônio Artur de Souza, Márcio Noveli, Ítalo Fernando Minello, João Otávio Montanha Endrici, Ewerton Alex Avelar

\section{METODOLOGIA}

O principal objetivo desta pesquisa foi analisar os sistemas de informações utilizados pelas EPEs para auxiliar nas tomadas de decisão referentes aos complexos processos de EC e FP. Para atingir tal objetivo, foi necessário realizar um profundo estudo das peculiaridades do processo produtivo dessas empresas, das metodologias usadas para a EC e FP, e dos sistemas de informações utilizados pelas empresas. Diversos profissionais e acadêmicos de diferentes níveis trabalharam na pesquisa, trocando experiências, tentando compreender as peculiaridades das EPEs no que se refere aos processos de EC e FP, e analisando os sistemas utilizados pelas empresas para ajudar nas decisões referentes aos mesmos.

A pesquisa consistiu de estudos de casos de natureza exploratória e qualitativa. Foi realizada em EPEs da região noroeste do Paraná, no período de 2002 e 2004. Visou estudar os processos de EC e FP e avaliar os sistemas de informações para suporte às tomadas de decisão utilizados nesses processos. Para Yin (2005), o estudo de caso é a forma de se fazer pesquisa empírica, investigando fenômenos contemporâneos dentro de seu contexto de vida real, em situações em que as fronteiras entre o fenômeno e o contexto não são claramente estabelecidas, onde se utilizam múltiplas fontes de evidência. De acordo com Malhotra (2003), a pesquisa exploratória descritiva qualitativa proporciona melhor visão e compreensão do contexto do problema.

Primeiramente, foi realizada uma intensa pesquisa bibliográfica, com o objetivo de identificar e selecionar as principais publicações relacionadas com o tema da pesquisa, tanto em nível nacional como internacional, para o seu estudo. Em seguida, foi realizado um estudo dos mais relevantes e recentes livros, artigos, teses, dissertações e outras publicações de áreas afins (marketing, microeconomia, produção, etc), no sentido de ampliar o conhecimento e identificar resultados de pesquisas nessas áreas.

Posteriormente, várias empresas foram contatadas e convidadas a colaborar com a pesquisa. Essa colaboração seria no sentido de permitir (1) entrevistas com seus 
Análise de sistemas de informações utilizados como suporte para os processos de estimação de custos e formação de preços

Antônio Artur de Souza, Márcio Noveli, Ítalo Fernando Minello, João Otávio Montanha Endrici, Ewerton Alex Avelar

estimadores de custos e tomadores de decisão das áreas de custos, de estabelecimento de preços de venda e de controle e planejamento da produção; e (2) análise dos sistemas de informações para suporte à decisão utilizados por esses profissionais. Para identificar as empresas que potencialmente poderiam participar da pesquisa, realizou-se uma consulta na Junta Comercial do Paraná (JUCEPAR), e criouse um banco de dados das empresas, o qual permitiu uma escolha mais objetiva e eficiente daquelas que, preferencialmente, poderiam ser objeto de estudo na pesquisa. Foram estudadas 12 EPEs, de diferentes setores, durante a pesquisa.

A coleta de dados foi realizada por meio de entrevistas e questionários semiestruturados aplicados aos profissionais responsáveis pelos processos de EC e ou FP nas EPEs selecionadas. Estes questionários tinham o objetivo de verificar como as EPEs analisam, obtêm e tratam seus custos, e, também, qual a influência que eles exercem na tomada de decisões de preços de seus produtos. Também objetivava identificar as ferramentas computacionais usadas por elas para dar suporte aos processos de EC e de FP e suas necessidades com relação aos SSDs nas áreas de EC, estabelecimento de preços de venda e planejamento e controle da produção. Foram enviados 70 questionários para uma amostra de empresas com as quais, não foi possível o encontro direto com os profissionais.

As entrevistas foram realizadas com a finalidade de analisar e compreender as metodologias usadas pelas empresas nos processos de EC e FP, bem como de identificar fatores internos e externos que afetam decisões referentes a esses processos, coletando o maior número de informações possível. Durante as entrevistas, os entrevistadores, de posse de um roteiro com questões abertas, tiveram liberdade para questionar as razões e os motivos procedimentais, direcionando a conversação para os assuntos que consideravam mais adequados, sem impor a limitação a uma estrutura formal. Foram realizadas entre 4 e 5 entrevistas com os profissionais de cada EPE estudada. Os resultados das entrevistas foram analisados qualitativamente com 0 objetivo de identificar deficiências e dificuldades dos processos de EC e FP.

Algumas entrevistas de complementação (follow-up) foram realizadas por e-mail e por telefone, com o objetivo de suprir a base de dados. Deve-se ressaltar a ocorrência 
Análise de sistemas de informações utilizados como suporte para os processos de estimação de custos e formação de preços

Antônio Artur de Souza, Márcio Noveli, Ítalo Fernando Minello, João Otávio Montanha Endrici, Ewerton Alex Avelar

de pesquisa documental, realizada com base em relatórios e documentos das empresas estudadas.

Para o estudo dos processos cognitivos (aquisição do conhecimento), empregouse a técnica verbal protocol analysis, que consiste em pedir aos entrevistados que verbalizem seus pensamentos, tais como ocorrem no momento em que formam uma decisão.

\section{OS PROCESSOS DE ESTIMAÇÃO DE CUSTOS E FORMAÇÃO DE PREÇOS EM EMPRESAS DE PRODUÇÃO POR ENCOMENDA}

A literatura disponível sobre EC e FP é bastante escassa, e comumente incompleta e esparsa, devido ao caráter superficial da maioria dos estudos. A pesquisa possibilitou mapear os diversos métodos usados para EC e FP nas EPEs, os fatores internos e externos que devem ser avaliados nesses processos, a difusão do uso de sistemas de informações para suporte à decisão nos mesmos e a avaliação de suas eficiências e deficiências.

A pesquisa teve como objeto de estudo EPEs do noroeste do Paraná. Muitas dessas empresas não elaboram produtos somente por ordem, pois já possuem clientes que demandam, de modo geral, seus produtos mensalmente. Isso faz com que parte da capacidade produtiva da empresa já esteja ocupada e o restante seja utilizado para atender a encomendas ocasionais.

Constatou-se na pesquisa, que são grandes as dificuldades enfrentadas pelas EPEs quando estimam seus custos e estabelecem o preço de venda de seu produto ou serviço, devido à natureza de suas operações. Constatou-se que o processo de elaboração de cada produto é minuciosamente analisado, pois os custos dependem muito do processo específico, e diferentes fatores precisam ser avaliados, pois, diversas vezes serão novos para a empresa, devido às especificações dos clientes. Constatou-se também que as dificuldades das EPEs na EC e na FP independem da participação dessas empresas no mercado e do volume de produção. Mesmo as 
Análise de sistemas de informações utilizados como suporte para os processos de estimação de custos e formação de preços

Antônio Artur de Souza, Márcio Noveli, Ítalo Fernando Minello, João Otávio Montanha Endrici, Ewerton Alex Avelar

empresas líderes do mercado têm dificuldades para tomar decisões corretas nesses processos.

A pressão competitiva decorrente da globalização é bastante agressiva e vem acirrando o mercado das EPEs nos últimos anos. Esse aumento da competitividade está forçando seus gerentes a investir continuamente na melhoria das metodologias de gestão utilizadas. Essas melhorias são no sentido de tentar tornar as operações mais eficientes, cortar custos desnecessários e atribuir o máximo de valor possível aos produtos.

Embora o mercado esteja cada vez mais competitivo, os gerentes das EPEs estudadas são, em geral, bastante otimistas em relação ao seu futuro. A maioria deles pretende fazer investimentos nas empresas para aumentar o faturamento. Além disso, expandir o mercado de atuação também faz parte das pretensões desses profissionais. Devido ao processo de globalização e ao aumento da competitividade no mercado das EPEs, os clientes exigem, cada vez mais, qualidade dos produtos e preços de venda compatíveis com os da concorrência. Embora a FP seja essencial para as EPEs sobreviverem no mercado, esse processo foi considerado demasiadamente complexo pelas empresas pesquisadas.

$\mathrm{Na}$ pesquisa constatou-se que a grande maioria dos profissionais utiliza o markup para estabelecer o preço de venda de seu produto nas EPEs, ou seja, é calculada uma margem de lucro fixa sobre o custo estimado. A principal diferenciação entre as metodologias utilizadas pelas empresas está na constituição do custo-base usado para a incidência da taxa de mark-up. Enquanto algumas empresas utilizam os custos plenos (soma dos custos e despesas da empresa) para incidir essa taxa, outras optam por utilizar apenas os custos marginais (custos acrescidos pelo produto a ser elaborado) como custo-base. O uso deste permite uma maior flexibilização para que as EPEs negociem com seus clientes; enquanto o uso dos custos plenos permite que todos os gastos da empresa sejam certamente recuperados no longo prazo.

Embora seja o método mais comumente usado pelas EPEs, a FP baseada nos custos apresenta falhas que devem ser ressaltadas. Primeiramente, com raras exceções, os custos dependem do volume produzido (economia de escala). Além disso, 
Análise de sistemas de informações utilizados como suporte para os processos de estimação de custos e formação de preços

Antônio Artur de Souza, Márcio Noveli, Ítalo Fernando Minello, João Otávio Montanha Endrici, Ewerton Alex Avelar

uma empresa não poderá precificar o seu produto acima do valor percebido pelo consumidor. Ou seja, quem, em última instância, define o limite máximo do preço é o consumidor e não os custos.

Os tomadores de decisão das EPEs pesquisadas afirmaram que o preço varia, principalmente, em função de três fatores: custos, concorrentes e clientes. Essa constatação da pesquisa valida a afirmação da maioria dos autores que constituíram a base teórica da mesma, os quais ressaltavam esses três fatores como os mais importantes para a FP.

Entretanto, observou-se que o grau de importância para cada um desses fatores varia muito entre as EPEs pesquisadas.

Assim que recebem um pedido para a fabricação de um produto, os gerentes das EPEs estimam e avaliam todos os custos necessários à sua fabricação. Caso os custos sejam demasiadamente altos para a empresa (caso seja necessário comprar novas máquinas, por exemplo), ela recusará o pedido, uma vez que o preço que deverá ser estabelecido será acima do aceitável.

Analisar os concorrentes é essencial. Oferecer um preço incompatível com o da concorrência fará com que as EPEs percam pedidos de fabricação. Avaliar o cliente é outra tarefa fundamental. Necessita-se levar em consideração diversos aspectos, entre eles: o potencial de futuros negócios que podem ser gerados com o cliente e o seu interesse no produto.

Independentemente do método a ser utilizado, o processo de FP estará sempre vinculado ao processo de $\mathrm{EC}$, e ambos estarão sujeitos a fatores, internos e externos à empresa, que deverão ser avaliados pelos tomadores de decisão para que possam ser formados preços mais condizentes possíveis com a realidade operacional e mercadológica do produto a ser fabricado.

Conhecer os próprios custos também é crucial para as EPEs. Ignorá-los ou relegá-los a segundo plano é ameaçar a prosperidade da empresa. Essa importância dos custos é reconhecida pelos gerentes das EPEs, nas quais, mais de $80 \%$ dos profissionais entrevistados se preocupam em estimar os custos o mais próximo possível 
Análise de sistemas de informações utilizados como suporte para os processos de estimação de custos e formação de preços

Antônio Artur de Souza, Márcio Noveli, Ítalo Fernando Minello, João Otávio Montanha Endrici, Ewerton Alex Avelar

do real, sendo que alguns acreditam que a EC é uma das atividades mais importantes a ser executada por este tipo de empresa.

Essa atenção dispensada aos custos pelos profissionais nas EPEs é justificável, visto que eles influenciam diversas decisões nessas empresas, muitas delas extremamente importantes para o seu futuro. Além da FP, como já foi exaustivamente avaliado ao longo deste trabalho, os custos influenciam várias outras decisões nessas empresas. As principais identificadas pela pesquisa foram: terceirização de serviços ou segmento da produção, lançamento de novos produtos, cancelamento (descontinuidade) de produtos não rentáveis, ampliação da capacidade produtiva, estabelecimento de parcerias, prorrogação de contratos e compra de materiais e máquinas.

Diversos métodos são utilizados no processo de EC nas EPEs. A maioria dos estimadores entrevistados faz uso de sistemas de custeio tradicionais (custeio por absorção, custeio direto e $A B C$ ) para estimar seus custos. Porém, uma parte das empresas pesquisadas não utiliza um método formal para a EC. Elas utilizam metodologias propostas por profissionais que acreditam que sejam mais adaptadas à realidade de sua empresa especificamente.

Além disso, o know how dos profissionais, principalmente na EC, é visto como de primordial importância pelas empresas. É unânime entre os estimadores entrevistados, a carência de ferramentas mais adequadas para conduzirem suas tarefas relativas à EC e FP.

Os custos fixos (custos que não variam de acordo com o volume de produção) são vistos com bastante preocupação pelos tomadores de decisão nas EPEs. Eles compreendem que a ociosidade é mais prejudicial do que a margem de lucro zero. Alguns dos profissionais entrevistados afirmaram que, às vezes, quando o volume de produção é baixo, são aceitos trabalhos sem nenhuma margem de lucro, com a finalidade de cobrir os custos fixos.

Nas EPEs, a necessidade por informações relativas ao processo de EC mobiliza os departamentos que detêm essas informações para que as tornem disponíveis para o tomador de decisão. A partir dos dados obtidos na pesquisa, foi possível mapear os 
Análise de sistemas de informações utilizados como suporte para os processos de estimação de custos e formação de preços

Antônio Artur de Souza, Márcio Noveli, Ítalo Fernando Minello, João Otávio Montanha Endrici, Ewerton Alex Avelar

departamentos que detêm essas informações e quem as utilizam: o setor de Produção é percebido como a principal fonte de informações de custos para os tomadores de decisão nas EPEs. Entretanto, estes custos são relacionados principalmente a materiais diretos, o que, dentro da problemática observada, não contribui de forma significativa, pois os custos diretos são alocados diretamente aos produtos. Isso se traduz em custeamento, sem a necessidade de rateios arbitrários ou enviesados, que são problemas discutidos neste trabalho.

Verificou-se que os setores de Planejamento e Controle fornecem informações sobre custos indiretos de fabricação. Nestes setores, deve-se verificar a possibilidade de vieses ou de arbitrariedades na definição de parâmetros para a estimação dos custos indiretos. Em relação à utilização das informações, o setor de Planejamento (31\%) é o que mais utiliza dados de custos, seguido pelo de Produção (27\%).

Assim, conhecer os custos e as variáveis que o afetam é fundamental para as EPEs, principalmente, no atual ambiente instável e de acirrada competição. Portanto, o primeiro passo para que essas empresas consigam longevidade no mercado consiste em promover um profundo estudo dos próprios custos.

Durante os processos, tanto de EC como de FP, uma diversidade de fatores que os influenciam diretamente devem ser avaliados. Eles estão presentes tanto no ambiente empresarial quanto fora dele e devem ter sua influência estudada constantemente. Mesmo com os dados mais acurados possíveis sobre custos, a negligência da análise desses fatores fará com que a EC e a FP sejam distorcidas.

\section{FATORES INTERNOS E EXTERNOS E A INTERDEPENDÊNCIA ENTRE ESTIMAÇÃO DE CUSTOS E FORMAÇÃO DE PREÇOS}

Diversos fatores influenciam os processos de EC e FP em EPEs. Eles devem ser bem analisados pelos tomadores de decisão para que não sejam cometidos equívocos durante esses processos. Tais fatores podem ser divididos em internos e externos à empresa. 
Análise de sistemas de informações utilizados como suporte para os processos de estimação de custos e formação de preços

Antônio Artur de Souza, Márcio Noveli, Ítalo Fernando Minello, João Otávio Montanha Endrici, Ewerton Alex Avelar

Segundo De Souza et al. (2000), os fatores podem ser vistos como uma série de propriedades ou condições do ambiente que influenciam a tomada de decisão. Os fatores internos influenciam os custos de determinados produtos e são calculados com base nas informações internas da empresa. Os fatores externos afetam direta e indiretamente a EC e a FP. Eles exigem maior esforço no seu acompanhamento por parte dos tomadores de decisão por estarem fora da organização e não no ambiente de trabalho do profissional.

Durante a pesquisa, foram identificados 40 fatores (17 internos e 23 externos) que devem ser analisados pelos profissionais nas EPEs, em cada uma de suas decisões, para que sejam executadas coerentemente. Para um melhor entendimento dos fatores e da influência que exercem nos processos de EC e FP, optou-se por separá-los em quatro grupos (Quadro 1): Relacionados aos produtos e Relacionados à empresa (internos); Relacionados aos clientes e Relacionados ao mercado (externos).

\begin{tabular}{|l|l|}
\hline \multicolumn{1}{|c|}{ Fatores Internos } & \multicolumn{1}{|c|}{ Fatores Externos } \\
\hline \multicolumn{1}{|c|}{ Relacionados aos produtos } & \multicolumn{1}{c|}{ Relacionados aos clientes } \\
\hline $\begin{array}{l}\text { Existência do produto a ser fabricado, potencial de } \\
\text { futuros negócios, risco de acidentes, ciclo de vida } \\
\text { do produto, complexidade do produto, matéria- } \\
\text { prima utilizada, problemas de setup, know how e } \\
\text { feeling. }\end{array}$ & $\begin{array}{l}\text { Confiabilidade em termos das especificações } \\
\text { apresentadas pelos clientes, interesse no produto, } \\
\text { forma de seleção de fornecedores, principal } \\
\text { preferência do cliente (preço, qualidade ou prazo } \\
\text { de entrega), confiabilidade do cliente, } \\
\text { relacionamentos com clientes antigos, potencial } \\
\text { para futuros negócios, data e prazo de entrega, } \\
\text { negociação e poder aquisitivo dos clientes. }\end{array}$ \\
\hline \multicolumn{1}{|c|}{ Relacionados à empresa } & \multicolumn{1}{c|}{ Relacionados ao mercado } \\
\hline $\begin{array}{l}\text { Situação financeira (baseada no orçamento), } \\
\text { capacidade produtiva, participação de um dado } \\
\text { projeto na produção total, dependência do } \\
\text { fornecedor, estratégia de negócios, espaço físico, } \\
\text { disponibilidade de cada setor da empresa e mão- } \\
\text { de-obra qualificada. }\end{array}$ & $\begin{array}{l}\text { Concorrência do pedido, potencial de se entrar em } \\
\text { um novo setor de mercado, expansão do mercado, } \\
\text { frete, local de instalação, juros, globalização, } \\
\text { variaço cambial, greves, intempéries climáticas, } \\
\text { medidas governamentais. }\end{array}$ \\
\hline
\end{tabular}

Quadro 1. Fatores internos e externos que influenciam os processos de EC e FP

A pesquisa nas EPEs evidenciou a interdependência entre a EC e FP, uma vez que utilizando o mark-up para estabelecer seu preço final a EC serve de referência e base de cálculo para a FP. Porém, a EC também sofre influência direta da FP, pois alguns fatores, como a concorrência do pedido, exigem que a EC seja a mais real possível, de modo a traçar os limites de preço que a empresa poderá utilizar para 
Análise de sistemas de informações utilizados como suporte para os processos de estimação de custos e formação de preços

Antônio Artur de Souza, Márcio Noveli, Ítalo Fernando Minello, João Otávio Montanha Endrici, Ewerton Alex Avelar

conquistar o cliente sem incorrer em prejuízos, uma vez que o principal objetivo da EC é estabelecer a relação entre os custos e os fatores que os afetam. Pode-se observar que os fatores internos e externos incidem igualmente tanto sobre a EC como sobre a FP. Contudo, seu grau de importância vai variar de empresa para empresa, segundo a maneira como consideram mais relevantes ou os fatores internos ou externos ao seu negócio/ramo de atividade.

Uma vez que os fatores internos e externos afetam diretamente os processos de EC e FP e, consequentemente, a tomada de decisão, o tratamento e a análise desses fatores visando prever suas consequências nas atividades da empresa será responsável em grande parte por seu desempenho no mercado. Esse acompanhamento/mapeamento dos fatores é uma tarefa complexa, devido ao número e à variedade de fatores que devem ser considerados. Essa variedade descarta a possibilidade de um modelo perfeito ou ideal de EC e FP, cabendo a cada empresa avaliar qual é o método que supre as suas necessidades de forma mais adequada em cada momento.

\section{SISTEMAS DE INFORMAÇÕES UTILIZADOS PARA SUPORTE À DECISÕES REFERENTES AOS PROCESSOS DE ESTIMAÇÃO DE CUSTOS E FORMAÇÃO DE PREÇOS}

Como já foi avaliado ao longo deste trabalho, as EPEs necessitam de informações confiáveis continuamente, para que seus gerentes possam tomar decisões coerentes e possam mantê-las competitivas. Assim, o uso de um bom sistema de informações nessas empresas é fundamental. O principal objetivo da pesquisa foi analisar os sistemas de informações utilizados pelas EPEs, para ajudar nas tomadas de decisão nos processos de EC e FP.

$\mathrm{Na}$ pesquisa constatou-se que a maioria das empresas utiliza algum sistema de informações computadorizado para auxiliar nas tomadas de decisão nos processos de EC e FP. O uso desse sistema tem por finalidade dar mais velocidade e credibilidade a esses processos. O porte da empresa é o principal fator influente na escolha do sistema 
Análise de sistemas de informações utilizados como suporte para os processos de estimação de custos e formação de preços

Antônio Artur de Souza, Márcio Noveli, Ítalo Fernando Minello, João Otávio Montanha Endrici, Ewerton Alex Avelar

de informações para suporte à decisão a ser utilizado. As grandes empresas utilizam sistemas específicos para auxiliar nesses processos. Por outro lado, as pequenas adaptam sistemas de baixo custo já existentes no mercado, tais como o Microsoft Access (MS-Access) e o Microsoft Excel (MSExcel).

Nas EPEs de grande porte são utilizados programas específicos para a EC e FP, e que integram toda a empresa. Devido a esta integração, é possível que cada centro de custo ou de atividades lance diretamente no sistema os seus gastos com produção. De maneira análoga, cada setor da empresa poderá coletar no sistema as informações que Ihe convém no que diz respeito à EC e à FP. Assim, os diferentes departamentos poderão consultar o sistema da mesma forma que a diretoria e os centros de atividades poderão requisitar as informações de que necessitam. Esses sistemas, em sua maioria, permitem a realização de análises históricas que são feitas com o intuito de comparar os resultados. Essas análises também são utilizadas para controle entre o custo estimado e o realmente incorrido na linha de produção.

Alguns sistemas utilizados pelas grandes empresas são corporativos, ou seja, para a manipulação dos dados deve-se migrá-los para outros sistemas, o que se faz com certa facilidade. Para isso, são utilizados alguns sistemas como o MS-Excel e o Microsoft Word.

Ao contrário das grandes empresas, as de pequeno porte utilizam programas mais simples, que são adaptados para servir às suas necessidades. Geralmente, são utilizadas planilhas do MS-Excel e banco de dados do MS-Access, os quais têm baixo custo de implantação, condizentemente com a disponibilidade financeira dessas empresas, e são adaptados internamente por elas próprias, de acordo com suas necessidades específicas.

Os dados nas pequenas empresas são coletados de forma manual pelos próprios funcionários, pelo gerente de produção ou pelo gerente administrativo. Depois, são repassados para uma só pessoa, geralmente a secretária, que fará a sua inserção no sistema. As informações geradas pelo sistema são transmitidas para uma pessoa que, sozinha, encarrega-se de tomar as decisões cabíveis no que se refere à EC e à FP. 
Análise de sistemas de informações utilizados como suporte para os processos de estimação de custos e formação de preços

Antônio Artur de Souza, Márcio Noveli, Ítalo Fernando Minello, João Otávio Montanha Endrici, Ewerton Alex Avelar

Embora a maioria das empresas utilize algum tipo de sistema de informações para auxiliar nas tomadas de decisão, seus gerentes, de modo geral, não dão muita relevância às informações geradas pelo sistema. Esse descrédito deve-se, possivelmente, às deficiências encontradas nesses sistemas, quando são utilizados para auxiliar nas tomadas de decisões referentes à EC e à FP nas EPEs. Usualmente, eles fornecem informações desnecessárias; outras vezes, não fornecem relatórios e informações relevantes para essas empresas, que devem ser conseguidos por meio de empresas terceirizadas. Isso ocorre devido ao fato de os sistemas utilizados não serem adequados para as EPEs, dada a flexibilidade que os processos de EC e FP demandam destes sistemas, em decorrência da particularidade do pedido de cada cliente.

O descrédito dos gerentes das EPEs em relação aos sistemas de informações utilizados para suportar decisões referentes aos processos de EC e FP provoca uma série de implicações nocivas a essas empresas. Uma vez que as informações geradas pelo sistema são relegadas a segundo plano, os profissionais utilizam o know how e o feeling para tomar suas decisões, descartando a possibilidade de usar métodos mais cientificamente fundamentados para isso. Além disso, diversos profissionais, devido ao descrédito em seus sistemas, permitem que estes sejam alimentados com informações e dados imprecisos, tornando-os falhos e aumentando sua deficiência no auxílio às tomadas de decisão.

Assim, uma ferramenta que poderia ser utilizada para aumentar a competitividade das EPEs, tem quase todo o seu potencial inexplorado. Essa afirmativa é comprovada pelos dados obtidos nas EPEs, que demonstram que tanto as empresas que utilizam algum sistema de informações, como aquelas que não fazem uso desse recurso, competem igualmente no mercado. Embora a tecnologia dos sistemas de informações tenha evoluído bastante nos últimos anos, as EPEs não têm utilizado o seu potencial para benefício próprio.

Neste sentido, Dazzi e Angeloni (2004) apresentam conclusões similares, afirmando que a comunicação, permitindo esclarecer os objetivos e as vantagens inerentes ao novo sistema, aplicáveis à organização e aos recursos humanos em 
Análise de sistemas de informações utilizados como suporte para os processos de estimação de custos e formação de preços

Antônio Artur de Souza, Márcio Noveli, Ítalo Fernando Minello, João Otávio Montanha Endrici, Ewerton Alex Avelar

particular. No entanto, a transparência dos processos comunicativos dentro das organizações pode ser prejudicada por causa das redes informais de comunicação, que são mais velozes e eficazes que as redes formais de comunicação.

\section{CONCLUSÃO}

$\mathrm{Na}$ pesquisa apresentada neste artigo, constatou-se a grande dificuldade enfrentada pelas EPEs no que se refere aos processos de EC e FP, devido ao peculiar processo produtivo que possuem, totalmente orientado pelas especificações dos clientes. Diante de toda a problemática enfrentada pelas EPEs nesses processos, questão crucial à sua sobrevivência, evidencia-se a necessidade do desenvolvimento de pesquisas que tratem desse tema específico. Ainda que os trabalhos não solucionem por completo a complexidade enfrentada pelas EPEs na EC e FP, qualquer avanço nesta área assume grande importância, diante da escassez de fundamentação científica, na qual os profissionais dessas empresas têm que tomar decisões.

O processo de FP nas EPEs é ainda mais complexo que nas demais empresas, devido à natureza de suas operações. Essas empresas têm de avaliar diversos fatores para estabelecer o preço de venda de seu produto, dentre os quais se destacam: custos, concorrentes e clientes. A FP nessas empresas é totalmente influenciada pelo processo de EC. Muitos dos produtos elaborados pelas EPEs são totalmente novos, e a principal referência para precificá-los são os custos necessários para a sua elaboração. Uma estimação imprecisa dos custos implica, necessariamente, um preço distorcido. $O$ método normalmente utilizado por essas empresas para estabelecer o preço de venda de seus produtos é o da utilização da taxa de mark-up. Este método não pode ser usado arbitrariamente sem a análise do volume de produção e do mercado no qual a EPE se insere.

Além de ser fundamental para o processo de FP, uma correta EC é essencial para as EPEs, uma vez que, estimados, os custos influenciam diversas decisões, muitas delas vitais para a longevidade dessas empresas, tais como: aumento da capacidade produtiva, lançamento de novos produtos e terceirização. Mesmo sendo 
Análise de sistemas de informações utilizados como suporte para os processos de estimação de custos e formação de preços

Antônio Artur de Souza, Márcio Noveli, Ítalo Fernando Minello, João Otávio Montanha Endrici, Ewerton Alex Avelar

essenciais para as EPEs, existem diversos empecilhos para uma correta EC, dentre os quais podem ser citados: o aumento dos custos indiretos nas indústrias (devido à mecanização da maioria dos processos) e a falta de implementação de novos sistemas depende de um processo claro e transparente de clareza nas informações disponíveis para o estimador, devido a deficiências nos sistemas de informações das empresas.

$O$ atual processo de EC nas EPEs requer do estimador um profundo conhecimento do processo industrial e do trabalho específico a ser realizado para a fabricação do produto, assim como conhecimento prático das discrepâncias entre os custos estimados e os efetivamente ocorridos, devido aos diferentes fatores que afetam a elaboração do produto.

Uma EC imprecisa levará a empresa a tomar decisões que não adicionam valor.

Foi possível identificar, com bastante acurácia, uma série de fatores que precisam ser avaliados durante o processo de tomada de decisão, por influenciarem decisivamente os processos de EC e FP. Esses fatores se dividem em fatores internos e externos à empresa, os quais devem ser analisados na elaboração de cada produto. Os fatores internos, embora estejam dentro do ambiente empresarial e sejam calculados com base em informações da própria empresa, são, diversas vezes, de difícil identificação e mensuração, devido a deficiências dos sistemas utilizados nessas empresas. Os fatores internos mais relevantes, segundo as empresas pesquisadas, foram: capacidade produtiva; disponibilidade de cada setor; riscos de acidentes; e situação financeira.

Os fatores externos exigem um esforço ainda maior no seu acompanhamento por parte dos gerentes, uma vez que não se encontram no ambiente de trabalho desses profissionais, mas possuem influência avassaladora sobre o mesmo. Dentre esses fatores, os apontados como mais importantes pelas EPEs analisadas foram: concorrência do pedido; medidas governamentais; interesse no produto; o potencial para futuros negócios; o tempo de entrega; mudanças na tecnologia; e o potencial de se entrar em um novo mercado.

Durante a EC e a FP, cada um dos fatores, tanto os internos como os externos à empresa, devem ter sua influência rigorosamente avaliada pelos tomadores de decisão 
Análise de sistemas de informações utilizados como suporte para os processos de estimação de custos e formação de preços

Antônio Artur de Souza, Márcio Noveli, Ítalo Fernando Minello, João Otávio Montanha Endrici, Ewerton Alex Avelar

em cada análise, já que, muitas vezes, são novos ou apresentam incertezas devido às especificações dos clientes.

$\mathrm{Na}$ análise dos sistemas de informações utilizados para auxiliar as tomadas de decisão nos processos de EC e FP, constatou-se uma deficiência dos mesmos em auxiliar os tomadores de decisão. Eles não são suficientemente dinâmicos para se adaptarem às necessidades exigidas pelo peculiar processo produtivo dessas empresas. As EPEs de menor porte utilizam sistemas simples adaptados para auxiliar nos processos de EC e FP nessas empresas, enquanto as empresas de maior porte utilizam sistemas específicos para esses processos. Em ambos os casos há deficiências nos sistemas, que não fornecem todas as informações necessárias.

A principal causa da deficiência desses sistemas de informações é o fato de eles não terem sido elaborados especificamente para lidar com as operações tão peculiares das EPEs. Os sistemas utilizados são adaptados. Mesmos nas empresas de grande porte, que utilizam sistemas específicos para EC e FP, estes não são flexíveis o suficiente para suprir as necessidades informacionais demandadas pelo peculiar processo produtivo dessas empresas. Portanto, mesmo constituindo-se em uma poderosa arma competitiva, os sistemas de informações não têm seu potencial utilizado pelas EPEs.

\section{REFERÊNCIAS}

ANTONELLO, C.S. 2004. A metamorfose da aprendizagem organizacional: uma revisão crítica. In: R. RUAS et al. Os novos horizontes da gestão: aprendizagem organizacional e competências. Porto Alegre, Bookman, 222 p.

ATKINSON, A.A. et al. 2000. Contabilidade gerencial. São Paulo, Editora Atlas, 812 p.

BRUNI, A.L. e FAMÁ, R. 2004. Gestão de custos e formação de preços com aplicações na calculadora HP 12C e Excel: inclui 150 exercícios resolvidos, a planilha CUSTOS.XLS e o conjunto de apresentações CUSTOS.PPT. 3aㅡ ed. São Paulo, Atlas, $551 \mathrm{p}$. 
Análise de sistemas de informações utilizados como suporte para os processos de estimação de custos e formação de preços

Antônio Artur de Souza, Márcio Noveli, Ítalo Fernando Minello, João Otávio Montanha Endrici, Ewerton Alex Avelar

DAZZI, M.C. e ANGELONI, M.T. 2004. Compreendendo o significado de gestão do conhecimento e a importância da comunicação em seu compartilhamento: um estudo de caso. In: ENANPAD, Curitiba, 2004. Anais... Curitiba. CD-ROM.

DE SOUZA, A.A.; AGUILAR, C.G. e NOGUEIRA, D.N. 2000. Fatores que influenciam a estimação de custos e formação de preços em empresas que fabricam sob encomenda. Contabilidade Vista e Revista, 11(2):21-25.

DE SOUZA, A.A. 1995. Developing a knowledge-based decision support system to aid make-toorder companies in cost estimation and pricing decisions. Lancaster, Inglaterra. Tese de doutorado. Department of Management Sciences, University of Lancaster, 386 p.

DOLAN, R.J. e SIMON, H. 1998. O poder dos preços: as melhores estratégias para ter lucro. São Paulo, Futura, 403 p.

KOTLER, P. 2000. Administração de marketing. 10ª ed., Rio de Janeiro, Prentice-Hall, $764 \mathrm{p}$.

KOTLER, P. e ARMSTRONG, G. 1999. Princípios de marketing. $7^{a}$ ed., Rio de Janeiro, Prentice-Hall do Brasil, $527 \mathrm{p}$.

LAWRENCE, P.R e LORSCH, J.W. 1972. O desenvolvimento de organizações: diagnóstico e ação. São Paulo, Edgard Blücher, 112 p.

MAHER, M. 2001. Contabilidade de custos: criando valor para a administração. São Paulo, Atlas, $905 \mathrm{p}$.

MALHOTRA, N.K. 2003. Pesquisa de marketing: uma orientação aplicada. São Paulo, Bookman, 720p.

PARENTE, J.G.O. 2003. Sistema de informação de marketing e a pesquisa de marketing. In: S.R. DIAS (org.), Gestão de marketing. São Paulo, Saraiva, 539 p.

SHIMIZU, T. 2001. Decisão nas organizações: introdução aos problemas de decisão encontrados nas organizações e nos sistemas de apoio à decisão. São Paulo, Atlas, $317 \mathrm{p}$.

SLACK, N.; CHAMBERS, S. e JOHNSTON, R. 2002. Administração da produção. 2aㅡ ed. São Paulo, Atlas.

TURBAN, E.; McLEAN, E. e WETHERBE, J. 2004. Tecnologia da informação para gestão: transformando os negócios na economia digital. $3^{3}$ ed., Porto Alegre, Bookman, $660 \mathrm{p}$. 
Análise de sistemas de informações utilizados como suporte para os processos de estimação de custos e formação de preços

Antônio Artur de Souza, Márcio Noveli, Ítalo Fernando Minello, João Otávio Montanha Endrici, Ewerton

Alex Avelar

YIN, R.K. 2005. Estudo de caso: planejamentos e métodos. 3a ed. São Paulo, Bookman, $212 \mathrm{p}$.

ZWASS, V. 1992. Management information systems. Dubuque, Wm. C. Brown Publishers, $928 \mathrm{p}$. 